\title{
Pseudoscalar Vertex and Quark Masses
}

\author{
Jean-René Cudell ${ }^{\mathrm{a}}$, Alain Le Yaouanc ${ }^{\mathrm{b}}$ and Carlotta Pittori ${ }^{a *}$ \\ ${ }^{a}$ Institut de Physique, Université de Liège au Sart Tilman, B-4000 Liège, Belgique. \\ b'L.P.T.H.E., Université de Paris Sud, Centre d'Orsay, 91405 Orsay, France.
}

\begin{abstract}
We analyse available data on the quark pseudoscalar vertex and extract the contribution of the Goldstone boson pole. The strength of the pole is found to be quite large at presently accessible scales. We draw the important consequences of this finding for the various definitions of quark masses.
\end{abstract}

\section{Introduction}

The expected behaviour of the quark pseudoscalar (PS) vertex in the continuum, near the chiral limit, can be described through a perturbative contribution plus a non-perturbative Goldstone boson contribution. According to the Wilson operator product expansion (OPE), the non-perturbative contribution must be power behaved, i.e. at large $p^{2}$ it must drop as $1 / p^{2}$ up to logs. On the lattice, the use of a non perturbative renormalisation scheme [1] makes this contribution manifest. Although it goes to zero for large momentum transfers, our purpose is to extract it from lattice simulation data, and to show that it is not negligible for presently accessible scales. Moreover, from the axial Ward identity (AWI), the forward PS vertex is directly related to the scalar part of the quark propagator. Hence in the study of propagator OPE, the Goldstone pole contribution in the PS vertex should corresponds to the dominant non-perturbative (power) contribution in the quark condensate. In the following we will show our recent results, presented in ref.[3], of the analysis of available lattice data on the quark pseudoscalar vertex. We have extracted the Goldstone contribution, in $1 / p^{2}$ and the strength of the pole is found to be quite large at presently accessible scales. We draw the important consequences of this finding for the various definitions of quark masses. Finally we will present some preliminary results of our work in progress [4 on the lattice quark propagator anal-

\footnotetext{
*Talk presented by Carlotta Pittori at LATTICE99
}

ysis.

\section{The quark PS vertex: results from the fit to lattice data}

In order to evaluate the non-perturbative Goldstone contribution, we have analised the QCDSF collaboration data [5], at $\beta=6.0$, for the bare one-particle-irreducible lattice PS vertex, $\Lambda_{5}$, presented through the product (see their fig.1)

$a m_{q} \tilde{\Gamma}_{5}\left(p^{2}\right) \equiv C \times a m_{q} \Gamma_{5}\left(q=0, p^{2}\right)$

at three $\kappa$ values, where $\Gamma_{5} \equiv \operatorname{Tr}\left(\gamma_{5} \Lambda_{5}\right) / 4$ and by construction, $C$ is a constant with the value $C=0.75$ in order to satisfy the AWI in a momentum range, increasing with $\kappa$.

As $\Gamma_{5}\left(q=0, p^{2}\right) \propto\left[A\left(p^{2}\right) / a m_{q}+B\left(p^{2}\right)\right]$, it seems reasonable to identify the $A\left(p^{2}\right)$ term as the Goldstone contribution and the second one as the perturbative contribution, if we are sufficiently close to the chiral limit. Let us fit the data to the form

$a m_{q} \tilde{\Gamma}_{5}\left(p^{2}\right)=A\left(p^{2}\right)+a m_{q} B\left(p^{2}\right)$

where, from the continuum Ward identity, one gets for the non-perturbative part:

$A\left(p^{2}\right)=A_{0} \times \frac{\left[\alpha_{s}\left(p^{2}\right)\right]^{7 / 11}}{a^{2} p^{2}}\left[1+22.0 \frac{\alpha_{s}\left(p^{2}\right)}{4 \pi}\right]$

and for the perturbative part:

$B\left(p^{2}\right)=B_{0} \times\left[\alpha_{s}\left(p^{2}\right)\right]^{4 / 11}\left[1+8.1 \frac{\alpha_{s}\left(p^{2}\right)}{4 \pi}\right]$

with $A_{0}$ and $B_{0}$ some constants. These two-loop renormalisation group improved corrections are 
valid for the MOM renormalisation scheme, in the Landau gauge. We shall first perform an extrapolation linear in $m_{q}$ of the three datasets to the chiral limit for each value of $p^{2}$. This fit gives us both $A\left(p^{2}\right)$ and $B\left(p^{2}\right)$.

The lattice data turn out to be quite close to the continuum theoretical expectations. Indeed one finds that:

- $A\left(p^{2}\right)$ is behaving remarkably close to $1 / p^{2}$ over a large interval of $p^{2}$ The Goldstone contribution appears to be very large: $a^{2} p^{2} A\left(p^{2}\right) \simeq 0.015$ from the lowest point $a^{2} p^{2}=0.16$. On the other hand, we do not see the log factors expected from the perturbative calculation.

- $B\left(p^{2}\right)$ is found to evolve in good conformity with the two-loop MOM renormalisation formula quoted above. We obtain $B_{0}=1.735$ which provides a very good fit to the data for $p^{2}$ larger than $2 \mathrm{GeV}$.

The Goldstone contribution is felt already at rather large quark masses and, for physical $u, d$ quarks it is in fact very large: $A\left(p^{2}\right)$ is larger than the perturbative part $a m_{q} B\left(p^{2}\right)$ even at rather large $p^{2}$, as shown in Fig. 1. This finding for the PS vertex has important consequences.

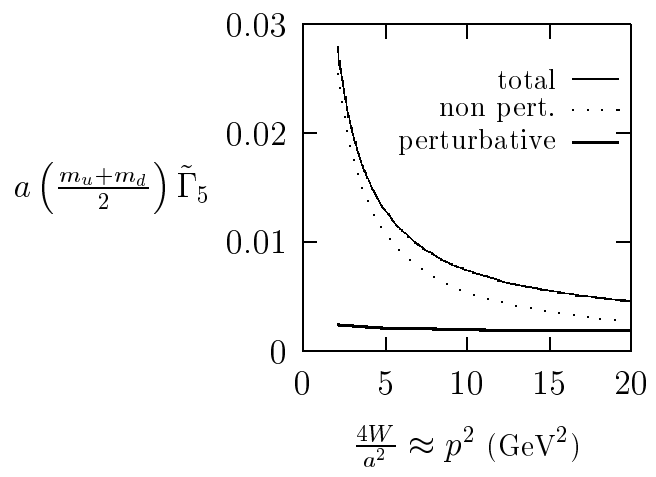

Figure 1. The value of $a m_{q} \tilde{\Gamma}_{5}$ for light quarks.

\section{A large Goldstone pole contribution: consequences on $Z_{P}^{M O M}$}

One can translate the above fit of $\Gamma_{5}$ into an expression for $Z_{P}^{M O M}$, or rather its inverse which is more directly physical:

$$
\frac{1}{Z_{P}^{M O M}\left(p^{2}\right)}=\frac{\Gamma_{5}\left(p^{2}\right)}{Z_{\psi}\left(p^{2}\right)}=\frac{A_{Z}\left(p^{2}\right)}{a m_{q}}+B_{Z}\left(p^{2}\right)
$$

This is to be contrasted with usual fits, which assume that $Z_{P}$ is linear in $a m_{q}$. The fact that the non-perturbative estimate of the full $Z_{P}$ differs sizeably from the short distance $B_{Z}$ already at the measured kappas, is a signal that it is not presently possible to work at $p^{2}$ high enough for the Goldstone contribution to be negligible. Hence, we must first subtract it from $Z_{P}^{-1}$.

$$
\left[Z_{P}^{S u b t r}\left(p^{2}\right)\right]^{-1}=\left[Z_{P}^{-1}\left(p^{2}\right)\right]-\frac{A_{Z}\left(p^{2}\right)}{a m_{q}}=B_{Z}\left(p^{2}\right)
$$

Numerically at $a^{2} p^{2}=1$ we find $Z_{P}^{S u b t r}=0.53$, which corresponds to the full resummed shortdistance contribution determined directly from the lattice data.

\section{Consequences on light quark masses}

To get an estimate of the consequences on the short distance MOM renormalised quark mass given by:

$\operatorname{am}_{A W I}^{\operatorname{Landau}}\left(\mu^{2}\right)=\rho \frac{Z_{A}}{Z_{P}\left(\mu^{2}\right)}$

where $\rho$ is a mass parameter, we use $Z_{P}^{S u b t r}$ and we find in the $\overline{\mathrm{MS}}$ scheme : $a m_{u, d}^{\overline{M S}} \sim 0.0024$, therefore about $4.6 \mathrm{MeV}$ at $N_{F}=0$. Note that these numbers are only indicative; in view of the many uncertainties in the subtraction procedure, we do not try to discuss the other sources of error necessary to give a real determination of the mass. Our aim is only to underline the necessity of the subtraction of the Goldstone contribution. This contribution, which is only parasitical and has to be subtracted in the calculation of $\overline{\mathrm{MS}}$ masses, retains an important physical meaning in other definitions of renormalised quark masses. Let us recall the Georgi-Politzer renormalisation condition for the quark propagator

$S_{R}^{-1}\left(p, \mu^{2}\right)=i \not p+m_{R}^{G P}\left(\mu^{2}\right) \quad$ at $\quad p^{2}=\mu^{2}$

This defines a renormalised quark mass $m_{R}^{G P}$ which can be related to the PS vertex through 
the axial Ward identity

$m_{A W I}^{\text {Landau }}\left(\mu^{2}\right) \Gamma_{5}^{R}\left(q=0, p^{2}, \mu^{2}\right)=\frac{\operatorname{Tr}\left[S_{R}^{-1}\left(p, \mu^{2}\right)\right]}{4}$

Numerically, using the full $Z_{P}\left(p^{2}\right)$, one gets $a m_{R}^{G P}\left(a^{2} p^{2}=1\right) \sim 0.018$, therefore around 34 $\mathrm{MeV}$ at $p=1.9 \mathrm{GeV}$, see Fig. 2. The magnitude is larger than what is expected from the quark condensate and from the perturbative evaluation of the Wilson coefficient, but in agreement with other physical expectations. We stress that, in

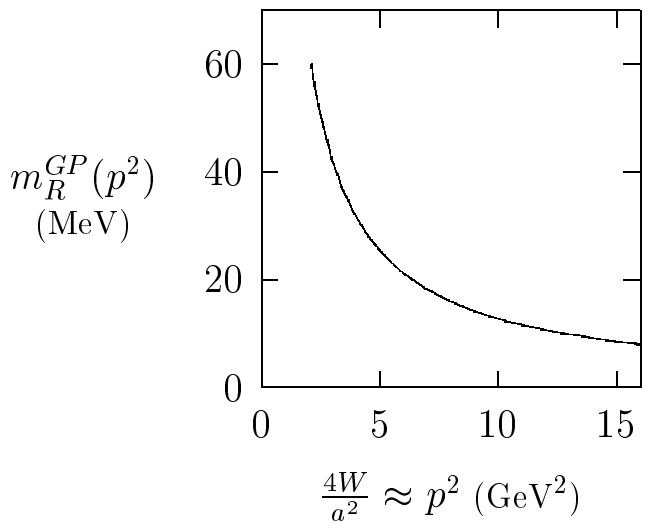

Figure 2. The value of the dynamical $u, d$ masses.

contrast to the standard $\overline{\mathrm{MS}}$ current mass, this mass does not vanish in the chiral limit, and it represents a dynamically generated mass for light quarks, though off-shell, gauge-dependent and Euclidean. This is a well-known signal of the spontaneous breakdown of the chiral symmetry.

\section{Relation with the lattice quark propa- gator analysis}

We have obtained analogous results directly by looking at the scalar part of the improved quark propagator. The discussion of the propagator lattice data involves delicate problems of improvement, since the $O\left(a^{2}\right)$ corrections appear to be dominant over the standard off-shell $O($ " $a$ ") improvement. One possible way out is to adopt an improved definition of the propagator as the solution of a non-linear equation which has a smoother tree-level $p^{2}$ dependence, see ref. [4]. In
Fig. 3 we present our preliminary results, not yet estrapolated to the chiral limit, for the ratio between the scalar and the vector part of the improved non-linear propagator, obtained by using the data of the APE collaboration [6]. The bare improved propagator is written as (here and in Fig. 3 the notation $A$ and $B$ do not correspond to the ones used in the previous sections),

$$
\left(S^{i m p}\left(p^{2}\right)\right)^{-1} \equiv A^{i m p}\left(p^{2}\right)(i \sin (a p))+B^{i m p}\left(p^{2}\right)
$$

hence in this case one has: $a m_{R}^{G P}\left(p^{2}\right)=$ $B^{i m p}\left(p^{2}\right) / A^{i m p}\left(p^{2}\right)$.

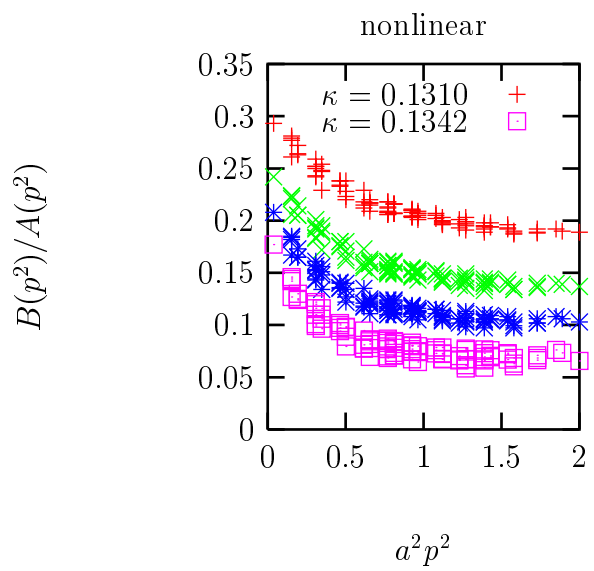

Figure 3. The value of $a m_{R}^{G P}\left(p^{2}\right)$ as obtained from the non-linear equation for the improved propagator.

\section{REFERENCES}

1. G. Martinelli et al., Nucl. Phys. B445 (1995) 81.

2. H. D. Politzer, Nucl. Phys. B117 (1976) 397.

3. J.R. Cudell, A. Le Yaouanc and C. Pittori, Phys. Lett. B454 (1999) 105.

4. J.R. Cudell, A. Le Yaouanc and C. Pittori, ULG-PNT-CP-99-1, in preparation.

5. Paul Rakow, for S. Capitani et al., Nucl. Phys. B (Proc. Suppl.) 63 (1998) 871.

6. G. Martinelli (APE collaboration), private comunication. 